\title{
Control of rotor-blade coupled vibrations using shaft-based actuation
}

\author{
René Hardam Christensen* and Ilmar Ferreira Santos \\ Department of Mechanical Engineering, Technical University of Denmark, DK-2800 Kgs. Lyngby, Denmark
}

\begin{abstract}
When implementing active control into bladed rotating machines aiming at reducing blade vibrations, it can be shown that blade as well as rotor vibrations can in fact be controlled by the use of only shaft-based actuation. Thus the blades have to be deliberately mistuned. This paper investigates the dynamical characteristics of a mistuned bladed rotor and shows how, why and when a bladed rotor becomes controllable and observable if properly mistuned. As part of such investigation modal controllability and observability of a tuned as well as a mistuned coupled rotor-blade system are analysed. The dependency of the controllability and observability on varying rotational speed and mode shape interaction phenomena between parametric and basis mode shape components are also analysed. Numerical results reveal a limitation of the achievable controllability and observability, once quantitative measures of modal controllability and observability converge toward steady levels as the degree of mistuning is increased. Finally, experimental control results are presented to prove the theoretical conclusions and to show the feasibility of controlling rotor and blade vibrations by means of shaft-based actuation in practice.
\end{abstract}

Keywords: Controllability, periodic system, bladed disc, periodic modal analysis

\section{Introduction}

The implementation of active vibration control into bladed rotating machines, such as for instance turbo machinery, implies that sensors and actuators will have to be built-in the blades monitoring their vibration levels and applying proper control action based on the measurements. Sensors can be built into the rotating blades to monitor their vibration levels, i.e. by using strain gages [4]. Alternative methods to measure the blade vibration levels by using sensors fixed in the non-rotating frame have also been developed to avoid sensors built into the blades, requiring signal transmission from a rotating to a non-rotating frame by using slip-rings or telemetric devices and also requiring high resistance to tough working conditions [18]. Building actuators into rotating blades is however much more complicated than implementing sensors. The only feasible actuation technique to be built-in for individual blade control is the use of smart materials, i.e. piezo-electric elements, which can also be used as sensing elements. However, it will be very costly and introduce many difficulties to be overcome if piezo-electric actuation/sensing elements should be embedded into each individual blade. Active controlled bearings for rotor vibration control have been developed for many years, applied for various purposes and shown their effectivenes in practical applications. Among the different types of active bearings are active magnetic bearings [8], active lubricated bearings [13], piezoactuated bearings [1] and bearings controlled by means of hydraulic actuators [16]. Therefore, one could ask: Can rotating blade vibrations also be controlled using such active controlled bearings? Are actuators built into the blades absolutely required in order to control rotor blade vibrations? Can the rotor as well as blade vibrations be monitored and controlled by means of shaft-based sensing and actuation solely?

Such questions have been studied by very few researchers. In the work of [11] the controllability of a bladed disk was analysed by studying its vibration mode shapes. It was shown that a tuned bladed disk cannot be controlled by

\footnotetext{
${ }^{*}$ Corresponding author: Ilmar Ferreira Santos, Department of Mechanical Engineering, Technical University of Denmark, Akademivej 404, DK-2800 Kgs. Lyngby, Denmark. Tel.: +45 452562 69; Fax: +45 4593 14 75; E-mail: ifs@ mek.dtu.dk.
} 
using only shaft-based actuation and sensing. However, if properly mistuned it was shown that the disk becomes controllable. Numerical control results of a mistuned disk controlled via shaft-based actuation were described in the work [11] and in $[9,10]$. The controllability analysis was based on a study of only time-invariant mode shapes while the time-variant vibration modes, known as parametric modes, which will occur due to vibration coupling between rotor and blades, were not considered. Therefore, in those cited works the blade vibrations are controlled by forcing the rotor/disk motion to minimize the blade deflections. If blade motion is coupled to rotor motion then blade vibration energy can be absorbed via controlling the motion of the rotor. Therefore, for bladed rotors presenting significant vibration coupling between rotor and blades such coupling and the presence of parametric coupled modes are very important to consider when analysing the system controllability. A methodology for studying the modal controllability and observability of a bladed disk, considering the rotor and blade vibration coupling, was presented in [19] and a tuned bladed disk was numerically analysed. Active control of a mistuned bladed rotor via shaft-based actuation was studied theoretically as well as experimentally in [20,21]. In those references the dynamic behavior of horizontal bladed rotors was focused, for example, wind turbines with heavy blades. These systems are subject to a significant period excitation due to gravity effect and blade weights, which may result in control problems. One of the experimental problems stated in $[20,21]$ was the reduction of control performance because considerable amount of the controller energy had to be used to deal with such periodic excitation. In this case more powerful actuators are needed to suppress parametric vibrations. Furthermore, no analyses of controllability and observability were carried out in [20,21], and such analyses turn out to be of fundamental importance. Due to the presence of the blade stiffening effect, which leads to changes of blade natural frequencies, and interaction among basic and parametric vibration modes, the controllability and observability are, in fact, strongly dependent on angular velocity. It means that a bladed rotor with a strong dynamic coupling among rotor and blades, which is controllable when operating at a given angular velocity, not necessarily will be also controllable when operating at another angular velocity.

In this framework the present paper is a natural continuation of the research works [20,21]. This paper is though devoted to vertical bladed rotors in which the periodic excitation due to gravity and blade weights does not play a significant role. From the theoretical viewpoint the main contributions are: i) the modal controllability and observability and their dependency on stiffening and frequency veering effects [15] are carefully quantified in the absence of periodic excitation induced by the gravity; ii) it is shown how and why the introduction of deliberate blade mistuning makes the bladed rotor controllable and observable using only shaft-based actuation and sensing; iii) the influence of the rotational speed on the degrees of controllability and observability is investigated; iv) the influence of mistuning patterns on the controllability of vertical bladed rotors with different number of blades is also examined. Finally, the test rig was modified in order to test vertical bladed rotors. The main experimental contribution is: v) the verification of control performance improvement, when the controllers do not have to use their energy to deal with the periodic excitation due to the gravity and the blade weights. In other words, the feasibility of controlling blade vibrations in a vertical bladed rotors by means of shaft-based active control is tested, achieving significant better performances than those delivered by the controllers presented in [20,21].

\section{Rotor-blade system description and test facilities}

Figure 1(a) shows a schematic drawing of the considered actively controlled coupled rotor-blade system. Four flexible blades are radially attached onto a rigid disk/shaft supported by active controlled radial bearings. Tip masses are added to the blades to emphasize their inertia and the vibration coupling among rotor and blades. It is noticed that the movement of the rotor is assumed to be restricted into the $x y$-plane neglecting rotor angular movements and gyroscopic effects. Such assumption simplifies the mathematical model describing the dynamics of the system and for the theoretical analysis of this work, this will be a permissible assumption. However, small rotor angular movements can not be completely avoided in practical machines but the experimental test rig built for this study, shown in Fig. 1(b), is designed to minimize the influence of such effects. For practical use in real bladed rotating machinery rotor angular movements and gyroscopic effects may have to be considered. In this case, two additional degrees of freedom have to be added to the mathematical model. Geometrical and physical parameters characterizing the test rig are presented in Table 1. 
Table 1

Rotor and blade physical and geometrical properties

\begin{tabular}{llllllll}
\hline Rotor & & \multicolumn{7}{c}{ Blades } & & \\
\hline Mass & $m_{h x}$ & 10.5 & {$[\mathrm{~kg}]$} & Length & $L_{b i}$ & $80 \cdot 10^{-3}$ & {$[\mathrm{~m}]$} \\
& $m_{h y}$ & 8.6 & {$[\mathrm{~kg}]$} & Distributed mass & $\rho_{b i}$ & 0.195 & {$[\mathrm{~kg} / \mathrm{m}]$} \\
Stiffness $^{*}$ & $K_{h x}$ & $66 \cdot 10^{3}$ & {$[\mathrm{~N} / \mathrm{m}]$} & Elasticity & $E_{b i}$ & $2.0 \cdot 10^{11}$ & {$\left[\mathrm{~N} / \mathrm{m}^{2}\right]$} \\
& $K_{h y}$ & $77 \cdot 10^{3}$ & {$[\mathrm{~N} / \mathrm{m}]$} & Moment of inertia $(\mathrm{area})$ & $I_{b i}$ & $2.08 \cdot 10^{-12}$ & {$\left[\mathrm{~m}^{4}\right]$} \\
Damping $^{*}$ & $D_{h x}$ & $1.2 \cdot 10^{-6}$ & {$[\mathrm{~N} \cdot \mathrm{s} / \mathrm{m}]$} & Damping* & $D_{b i}$ & 0.8 & {$[\mathrm{~N} \cdot \mathrm{s} / \mathrm{m}]$} \\
& $D_{h y}$ & $1.5 \cdot 10^{-6}$ & {$[\mathrm{~N} \cdot \mathrm{s} / \mathrm{m}]$} & Tip mass & $m_{t i}$ & 0.109 & {$[\mathrm{~kg}]$} \\
Disk radius & $r$ & 0.04 & {$[\mathrm{~m}]$} & Tip mass moment of inertia & $J_{t i}$ & $3.35 \cdot 10^{-5}$ & {$\left[\mathrm{~kg} \cdot \mathrm{m}^{2}\right]$} \\
Disk mass & $m_{r}$ & 3.0 & {$[\mathrm{~kg}]$} & Tip mass length & $L_{t i}$ & $30 \cdot 10^{-3}$ & {$[\mathrm{~m}]$} \\
& & & & Blade location $(i=1,2,3,4)$ & $\alpha_{i}$ & $(i-1) \cdot \frac{\pi}{2}$ & {$[\mathrm{rad}]$} \\
\hline
\end{tabular}

*The damping and stiffness coefficients are experimentally identified from the test facilities.

The experimental test rig is composed of the four bladed rigid disk mounted in a flexible suspension to allow significant rotor lateral movement. The rotor is driven by an electro-motor transmitting its torque through a pulley and two flexible couplings. Inductive non-contact measurement probes are attached to the rig to monitor the rotor/shaft lateral motion. Strain gages are bonded onto each blade to measure their deflections. Analog signal conditioning electronic circuits are built into the rigid disk for amplifying and filtering the strain gage signals. The measured signals are transmitted from the rotating disk to a control unit through a slip-ring assembly. Two pairs of electromagnetic actuators are applied to act onto the flexible suspension to control the rotor lateral motion, working like an active bearing. Sensors and actuators are connected to a digital signal processor dSPACE DS-1103 PPC controller board hosted on a PC, for data acquisition and for running control algorithms.

\section{Mathematical modelling}

Due to the cyclic variation of the blade inertia distribution in time domain and the influence of centrifugal and coriolis effects, the general equations of motion for the coupled rotor-blade system are time-variant depending on rotor position, rotational speed and acceleration. For a details on the mathematical modelling and rotor-blade dynamical characteristics, see [12,19]. Rotating at constant speed the model complexity reduces to become periodic time-variant with rotational speed $\Omega$. Using the state-space formulation the equations of motion are written as:

$$
\begin{aligned}
\dot{\mathbf{x}}(t) & =\mathbf{A}(t) \mathbf{x}(t)+\mathbf{B}(t) \mathbf{u}(t)+\mathbf{F}(t) \\
\mathbf{y}(t) & =\mathbf{C x}(t)
\end{aligned}
$$

where $\mathbf{x}(t)$ is the vector of state variables, $\mathbf{u}(t)$ is the vector of control forces acting onto the structure, applied via the active bearings. $\mathbf{A}(t)$ denotes the periodic system matrix, $\mathbf{B}(t)$ the control input matrix and $\mathbf{F}(t)$ is a vector of internal forces caused by the presence of the centrifugal and coriolis effects. The output matrix $\mathbf{C}$ is defined so that the measured outputs $\mathbf{y}(t)$ are the shaft lateral position.

By using a periodic modal coordinate transformation, the state-space model Eqs (1) and (2) is transformed into a modal form, more suitable for system analysis purpose and controller design. Due to the time-periodicity of the system, a classical time-invariant modal coordinate transformation cannot be applied. Hence, a periodic modal transformation method will be used [17]. The efficiency of applying such modal transformation, not only for controller design purpose [22], but also for analysis of coupled rotor-blade systems and for mathematically to explain the presence of parametric vibrations of such systems were studied in [5].

The modal model consists of a system of independent equations of motion, where each equation represents one vibration mode. It is derived by introducing a vector of modal state variables $\boldsymbol{\xi}(t)$, defined by $\mathbf{x}(t)=\mathbf{R}(t) \boldsymbol{\xi}(t)$, where $\mathbf{R}(t)$ is the right modal matrix. Introducing this new vector of state variables, the system is rewritten into the form:

$$
\begin{aligned}
& \dot{\boldsymbol{\xi}}(t)=\mathbf{A} \boldsymbol{\xi}(t)+\mathbf{B}(t) \mathbf{u}(t)+\mathbf{F}(t) \\
& \mathbf{y}(t)=\mathbf{C}(t) \boldsymbol{\xi}(t)
\end{aligned}
$$




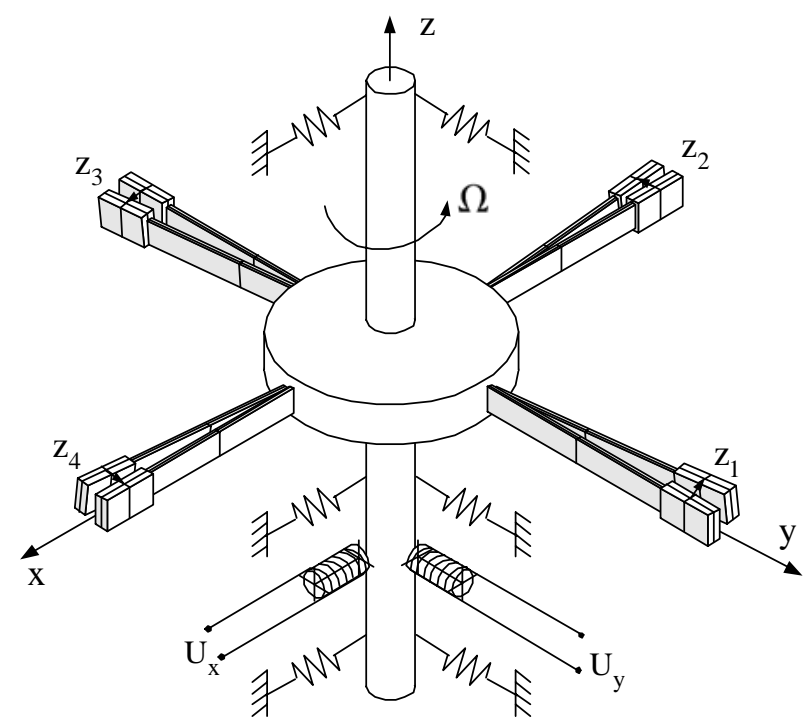

(a)

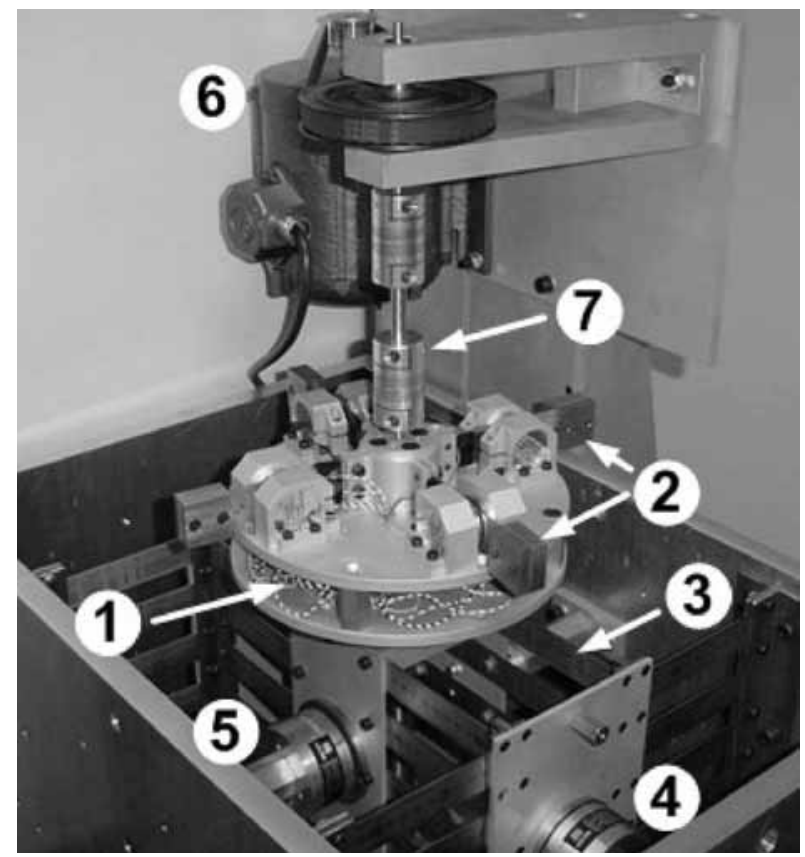

(b)

Fig. 1. Schematic drawing (a) showing the active controlled rotor-blade system and a photograph (b) showing the experimental test facilities. $\mathbf{1}$ rotor disk; $\mathbf{2}$ rotor blades; $\mathbf{3}$ flexible suspension; $\mathbf{4}$ electro-magnetic actuator $x$-direction; 5 electro-magnetic actuator $y$-direction; 6 AC-motor; 7 pulley and flexible couplings.

where $\mathbf{A}=\left[\mathbf{L}^{T}(t) \mathbf{A}(t) \mathbf{R}(t)-\mathbf{L}^{T}(t) \dot{\mathbf{R}}(t)\right]$ is a constant matrix containing the basis eigenvalues of $\mathbf{A}(t)$ along the diagonal, $\mathbf{B}(t)=\mathbf{L}^{T}(t) \mathbf{B}(t)$ is the periodic oscillatory modal control input matrix, $\mathbf{F}(t)=\mathbf{L}^{T}(t) \mathbf{F}(t)$ is the periodic oscillatory vector of modal forces and $\mathbf{C}(t)=\mathbf{C R}(t)$ is the periodic modal output matrix. 
The transformation matrices $\mathbf{R}(t)$ and $\mathbf{L}^{T}(t)$ denote the periodic complex right respectively left modal matrix of the system $\mathbf{A}(t)$. They are determined by solving a time-variant eigenvalue problem using Hill's method of infinite determinants [17]. Introducing the solution $\mathbf{x}(t)=\sum_{k=1}^{2 N} \mathbf{r}_{k}(t) \mathrm{e}^{\lambda_{k} t}$ into the equations of motion Eq. (1) yield an eigenproblem given by $\dot{\mathbf{r}}_{k}(t)+\left[\lambda_{k} \mathbf{I}-\mathbf{A}(t)\right] \mathbf{r}_{k}(t)=\mathbf{0}$ where $\lambda_{k}$ denotes the $k$ th eigenvalue associated to the right hand eigenvector $\mathbf{r}_{k}(t)$. Expressing the periodic terms of the matrix $\mathbf{A}(t)$ and the eigenvectors $\mathbf{r}_{k}(t)$ by means of Fourier series, the periodic eigenvalue problem is transformed into a time-invariant form. By solving this problem the eigenvectors are determined in terms of its Fourier expansion coefficients, i.e. $\mathbf{r}_{k}(t)=\sum_{j=-n}^{n} \mathbf{r}_{k, j} \mathrm{e}^{i j \Omega t}$. The right modal matrix is given by the form:

$$
\mathbf{R}(t)=\mathbf{R}(t+T)=\sum_{j=-n}^{n} \mathbf{R}_{j} \mathrm{e}^{i j \Omega t}
$$

where the Fourier coefficients are composed of the eigenvector coefficients $\mathbf{R}_{j}=\left[\mathbf{r}_{1, j}, \mathbf{r}_{2, j}, \ldots, \mathbf{r}_{2 N, j}\right]$. The modal matrix Fourier coefficients $\mathbf{R}_{j}$ represent basis and parametric vibration mode shape components of the system. The basis mode shapes, corresponding to the eigenvalues $\lambda_{k}$, are represented by $\mathbf{R}_{0}$ whereas parametric vibration mode shapes of order $j$ are given by $\mathbf{R}_{j}$, corresponding to the eigenvalues $\lambda_{k}+i j \Omega$.

It is worth to mention that because of the non-symmetric form of the matrix $\mathbf{A}(t)$ the right and left eigenvectors $\mathbf{r}_{k}(t)$ and $\mathbf{l}_{k}(t)$ are distinct, otherwise if $\mathbf{A}(t)$ had been symmetric $\mathbf{r}_{k}(t)$ and $\mathbf{l}_{k}(t)$ would be equal and $\mathbf{R}(t)=\mathbf{L}(t)$. The left modal matrix is determined so that the orthogonality relation is fulfilled $\mathbf{R}(t) \mathbf{L}^{T}(t)=\mathbf{I}$, that means it is written by the expansion $\mathbf{L}(t)=\sum_{j=-n}^{n} \mathbf{L}_{j} \mathrm{e}^{i j \Omega t}$.

\section{Measures of modal controllability and observability}

In order to check the ability of the active bearings and shaft position sensors to control and monitor the rotor and blade vibration levels, the controllability and observability are analysed. The modal controllability and observability can be analysed by checking the modal control input matrix $\mathcal{B}(t)$ and the modal output matrix $\mathcal{C}(t)$. General speaking, the system is, to some degree, modal controllable if no row of the modal control input matrix consists only of zeros and all modes are, to some degree, observable if no column of the modal output matrix consists only of zeros. Based on these facts, quantitative measures describing the degrees of controllability and observability of a specific mode, from all control actions and measurements can be deduced. Such measures are the so called gross measures of modal controllability and observability defined for time-invariant systems by [2]. Due to the time-variancy of the rotor-blade system, resulting in time-variant eigenvectors, measures of modal controllability and observability are for the actual system defined by the minimum values of such gross measures throughout one period of rotation, that is:

$$
\begin{array}{ll}
\mathrm{GMC}_{k}(t)=\min \left[\operatorname{norm}\left[\frac{\mathbf{l}_{k}^{T}(t) \mathbf{B}(t)}{\left\|\mathbf{I}_{k}^{T}(t)\right\|}\right]\right] \quad ; \quad t_{1} \leqslant t<t_{1}+\frac{2 \pi}{\Omega} \\
\mathrm{GMO}_{k}(t)=\min \left[\operatorname{norm}\left[\frac{\mathbf{C r}_{k}(t)}{\left\|\mathbf{r}_{k}(t)\right\|}\right]\right] \quad ; \quad t_{1} \leqslant t<t_{1}+\frac{2 \pi}{\Omega}
\end{array}
$$

where $\mathbf{r}_{k}(t)$ and $\mathbf{l}_{k}^{T}(t)$ are the $k$ th right and left eigenvector, respectively. Basically, these measures are obtained as the norms of the rows and columns of the modal input and output matrices normalized by the lengths of the eigenvectors. The measures are directly related to the angels between the eigenvectors and the rows/columns of the input and output matrices.

\section{Numerical results}

\subsection{Tuned system analysis}

Figure 2 presents waterfall diagrams showing system frequency responses as function of the rotational speed. Such responses are obtained by applying fast fourier transformation to numerical calculated time responses of the rotor 


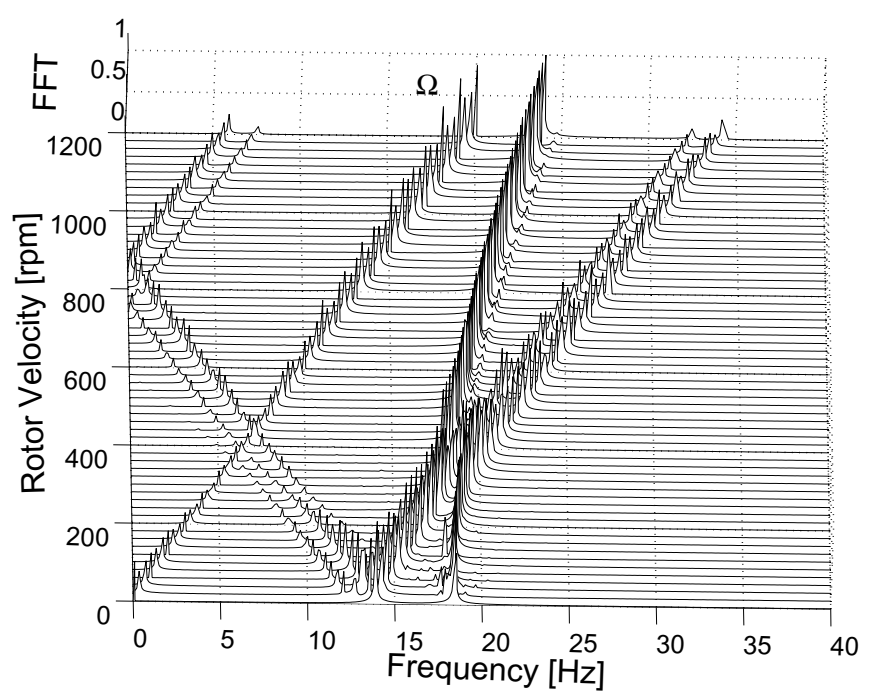

(a)

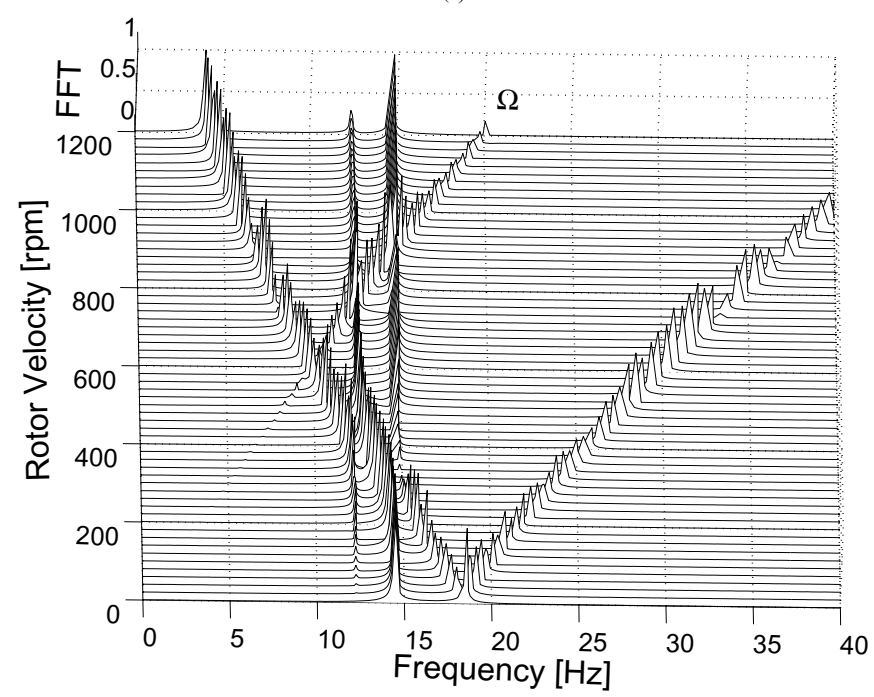

(b)

Fig. 2. Waterfall diagrams of the blade (a) and rotor movement (b) for the tuned rotor-blade system.

and blade movement, respectively. The diagrams clearly show the peculiarities characterizing the dynamics of this special kind of system. The presence of parametric vibration modes, due to the vibration coupling among the rigid disk/shaft and the flexible blade motions [12], is observed by the v-shaped frequency components. Figure 3 shows the frequency components present in the waterfall diagrams and the relation of each component to its respective vibration mode. The number denote the number of the specific mode and the letter $\mathrm{M}$ denotes basis modes while $\mathrm{P}, \mathrm{R}$ and $\mathrm{V}$ denote parametric mode components. For instance, the components $\mathrm{R} 1$ and $\mathrm{R} 2$ are backward rotating contributions of order 1 from the rotor natural frequencies to the blade movement while P1 and P2 are forward rotating contributions of order 1 to the blade movement. The components R5 and P6 are backward respectively forward rotating contributions of order 1 from the blade natural frequency to the rotor movement. The component V5 is a backward rotating parametric mode shape component of order -2 . The centrifugal stiffening is observed by the increasing blade eigenfrequencies with rotational speed, see M3, M4, M5 and M6 in Fig. 3. The remaining frequency components M1 and M2 are the disk/shaft lateral movement natural frequencies in the $x$ and $y$ direction, 


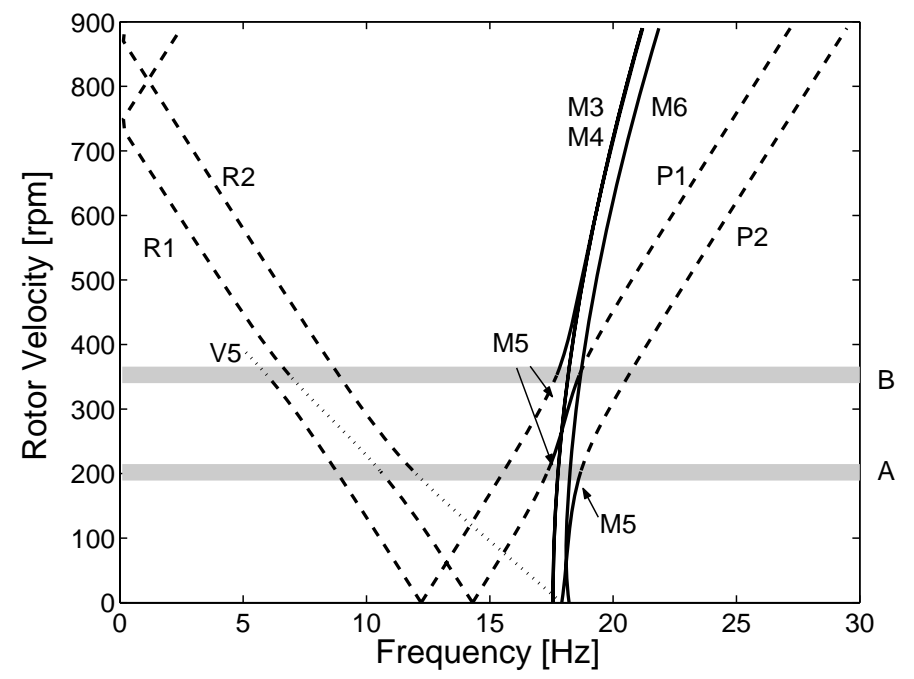

(a)

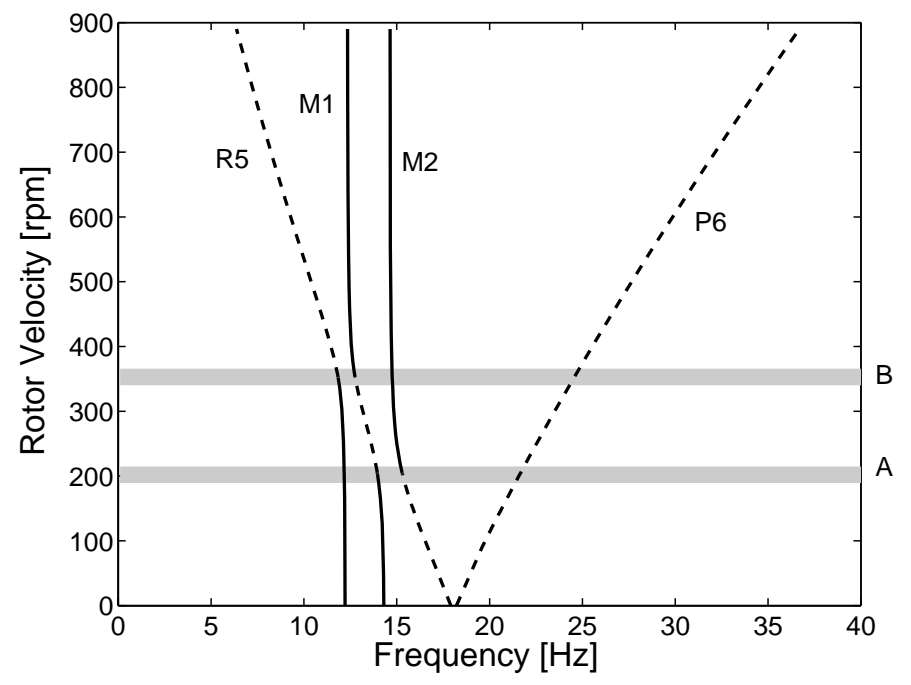

(b)

Fig. 3. Basis (-) and parametric eigenvalues of order $\pm 1(--)$ and of order $-2(\ldots)$ for the blade (a) respectively rotor movement (b).

respectively.

Due to the velocity dependency of the frequencies of the parametric mode components, these will at certain velocities interact with the basis mode components. The parametric mode shape components and the basis mode components tend to converge towards each other at the velocities $\sim 200 \mathrm{rpm}$ and $\sim 350 \mathrm{rpm}$ denoted as transition regions (A) and (B). At these specific angular velocities the natural frequencies of the system and the mode shapes will converge, interact and then veer apart without crossing. Such phenomenon, known as frequency veering [15], can be detected among the basis modes and the parametric mode components of order \pm 1 and -2 . Due to this frequency veering, the natural frequency of the basis mode component denoted by M5 will not, in fact, correspond to the fifth mode at all velocities. Dependent on the specific angular velocity it will, in fact, be the third, fifth or sixth mode. Though, throughout this study this particular mode will be denoted as the fifth mode, related to its mode number of the non-rotating system.

As it was theoretically explained by the modal model and observed in the Figs 2 and 3, the vibration mode shapes 


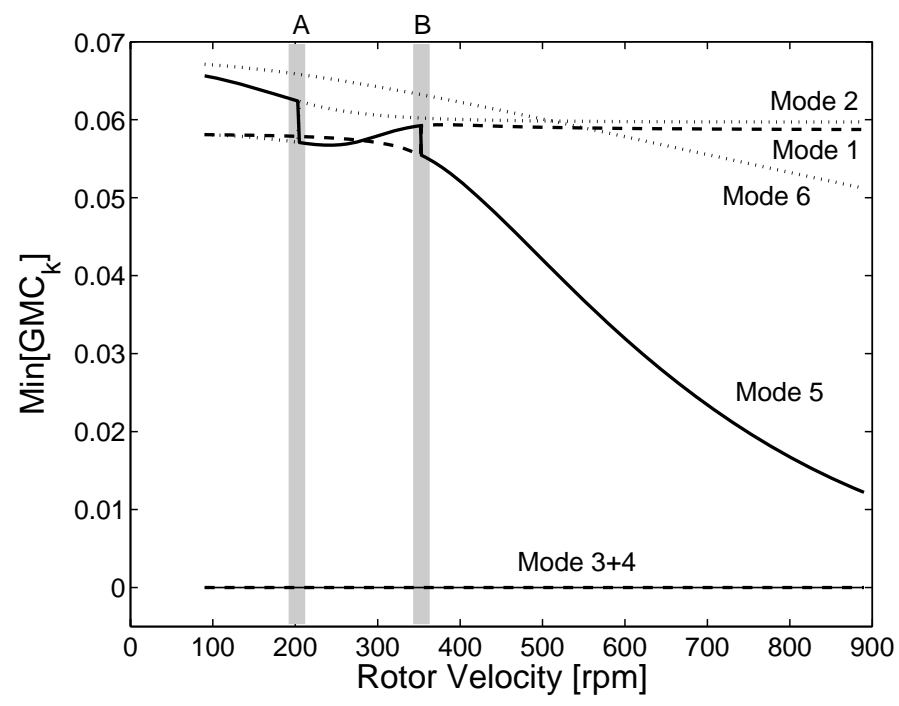

(a)

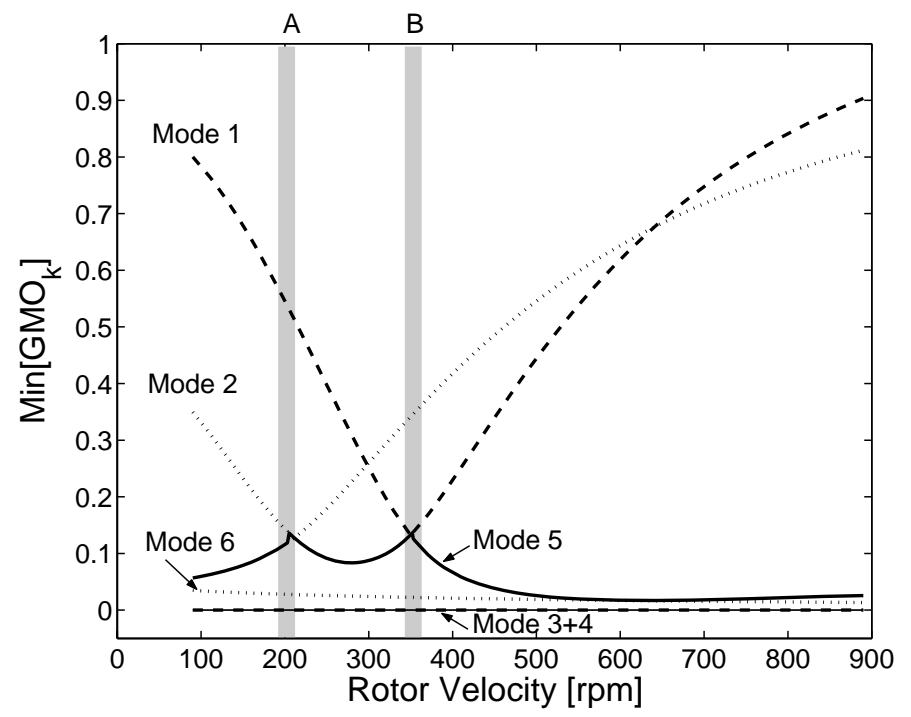

(b)

Fig. 4. Indices of modal controllability (a) and observability (b) for the tuned rotor-blade system.

vary with rotational speed, due to the centrifugal stiffening and the parametric mode components. Consequently, the gross measures of modal controllability and observability, determined by using the time-variant eigenvectors, will vary as well. Figure 4 shows minimum values of the quantitative measures of modal controllability and observability through one period of rotation for the first six modes of the tuned rotor-blade system as functions of the rotational speed. The bladed disk is controlled and monitored by using only shaft-based actuation and sensing. The higher an index is, the more controllable or observable is the mode. If the index is zero then the mode is non-controllable or non-observable. The results show that if only shaft-based actuation and sensing are applied, only the modes 1 , 2, 5 and 6 can be controlled and observed. The modes 3 and 4 can neither be controlled nor be observed by using shaft-based actuation and sensing, once the controllability and observability indices of such modes are zero.

Observing the the controllability and observability indices in Fig. 4 it is worth to notice the indices during the mode shape crossing regions (A) and (B). At these particular regions the indices of the crossing modes, 2 crosses 


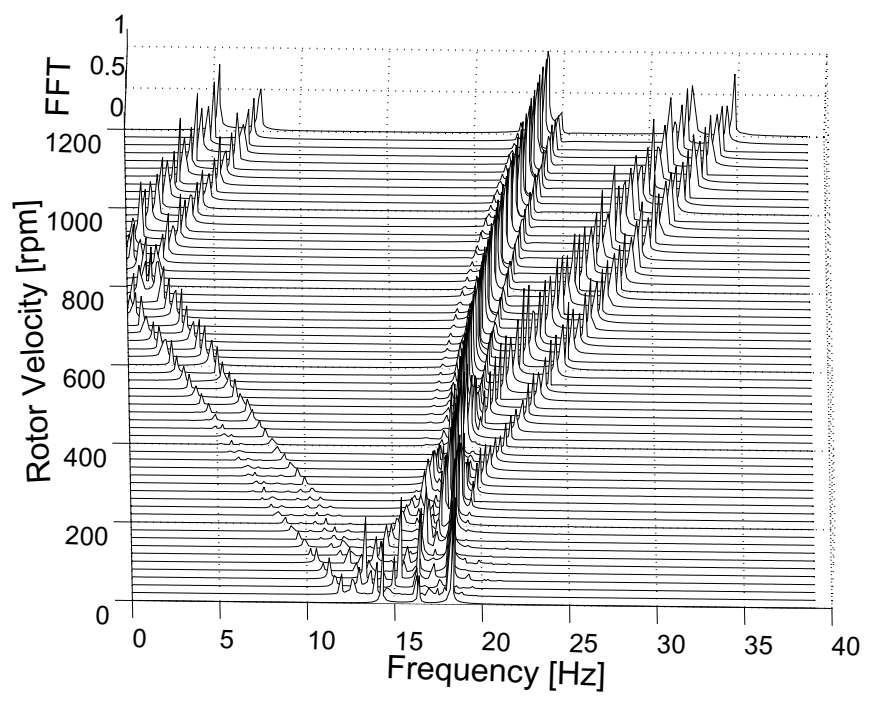

(a)

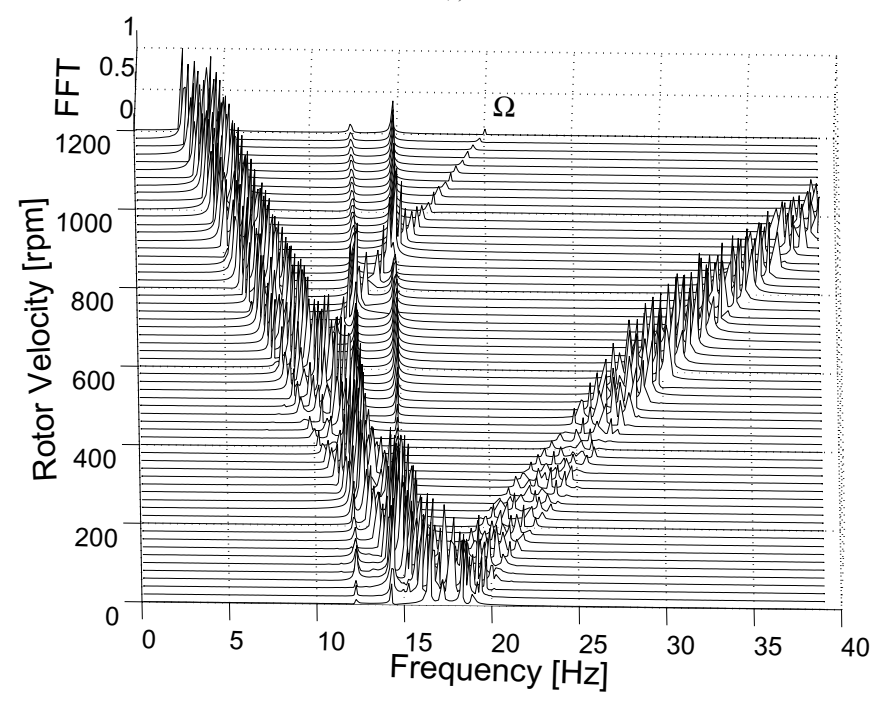

(b)

Fig. 5. Waterfall diagrams of the blade (a) and the rotor movement (b) for the mistuned rotor-blade system.

5 at (A) and 1 crosses 5 at (B), seems to converge towards similar values. More precisely, they converge as the velocity nears the transition velocity. The reason to this behaviour is, that the mode shapes when the velocities nears the transition phase tend to converge towards "identical" shapes. This can be observed by analysing the eigenvectors during the transition phase.

\subsection{Mistuned system analysis}

In the introduction it was described that bladed disk coupled vibrations can be controlled and monitored by using only shaft-based actuation and sensing, if the blades are properly mistuned. Blades can be mistuned by varying their modulus of elasticity, their length, their weight etc. Throughout this study the bladed disk is mistuned by varying the size of the blade tip masses. Onto the tip mass of blade number two is added $10 \%$ extra mass, to the third blade 


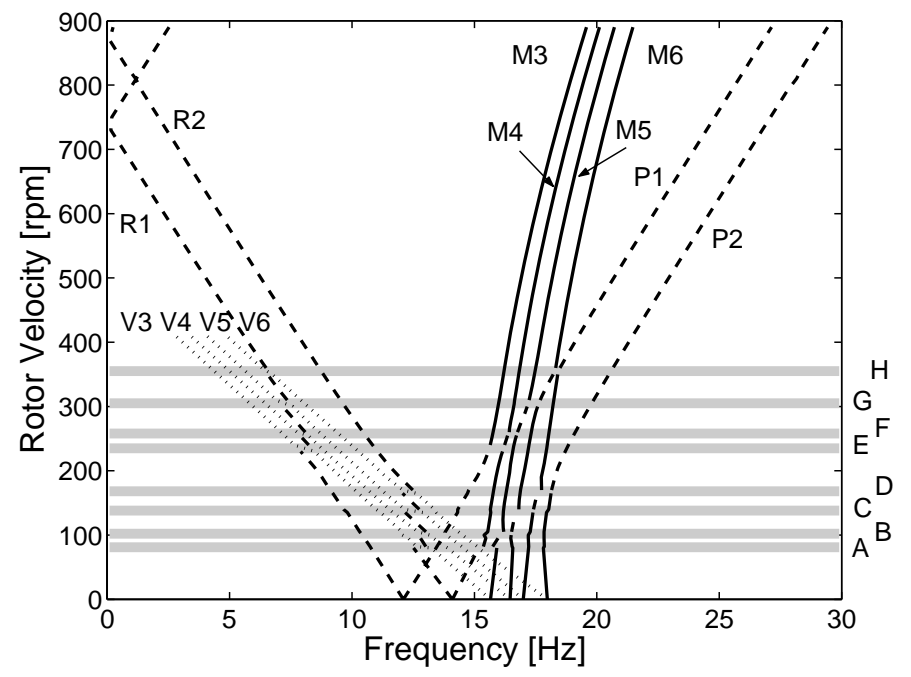

(a)

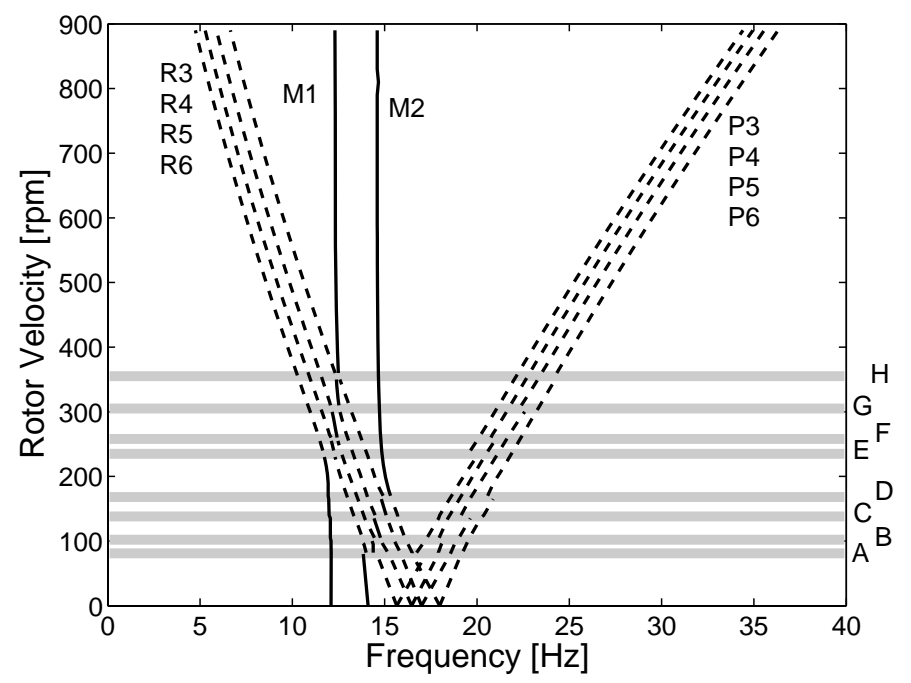

(b)

Fig. 6. Basis (-) and parametric eigenvalues of order $\pm 1(--)$ and of order $-2(\ldots)$ for the blade (a) respectively rotor movement (b) when the blades are deliberately mistuned.

$20 \%$ and to the fourth blade $30 \%$. The rotor unbalance such mistuning will introduce is compensated by adding extra balancing mass directly onto the disk.

Similar to the case of the tuned bladed disk, first, the dynamical properties and the influence of the mistuning are observed by numerical simulation. Figures 5 and 6 show waterfall diagrams and the relation of each frequency component to its respective mode. The most significant changes of the dynamical response caused by the introduction of deliberate mistuning, compared to the tuned system responses in Fig. 2, can be detected in the response of the rotor lateral motion. Figure 6 clearly shows that the blade mistuning results in an increased number of parametric mode components in the rotor movement, R3 to R6 and P3 to P6. Four forward rotating and four backward rotating mode components related to the motion of the blades are observed in the rotor motion. All four primarily blade related mode shapes become coupled to rotor lateral motion. This indicates that all primarily blade related modes might become detectable and controllable by monitoring and acting onto the rotor in the inertial frame by using an 
active bearing. A total of eight mode shape transition regions can be detected for the completely mistuned bladed disk resulting in an equal number of mode shape interaction regions at the speeds $81 \mathrm{rpm}(\mathrm{A}), 102 \mathrm{rpm}(\mathrm{B}), 138 \mathrm{rpm}$ (C), $168 \mathrm{rpm}$ (D), $235 \mathrm{rpm}$ (E), $258 \mathrm{rpm}$ (F), $305 \mathrm{rpm}(\mathrm{G})$ and $355 \mathrm{rpm}(\mathrm{H})$.

Figure 7 shows quantitative measures of modal controllability and observability as functions of the rotational speed for the deliberately mistuned system. The indices are only shown for angular velocities higher than $160 \mathrm{rpm}$ due to some difficulties related to the separation of basis and redundant solutions obtained when solving the time-variant eigenvalue problem for the system rotating at a low angular velocity. While the speed decreases the imaginary parts of the eigenvalues decrease as well. Moreover, the mistuning will distinguish the basis eigenvalues imaginary parts slightly. These factors imply that the basis eigenvalues of the system will tend to coincide with redundant eigenvalues making the separation process of the basis eigenvalues and vectors a very difficult task. Consequently, the controllability and observability indices of Fig. 7 are cut off at $160 \mathrm{rpm}$.

The limitation of the angular velocity at $160 \mathrm{rpm}$ hinders a complete analysis of the controllability and observability during the entire mode shape crossing region. However, the trends of the indices and the interaction among the basis and parametric mode shape components can be observed. As it was detected by the modal analysis, it is seen that all six modes are controllable by only shaft actuation, as a consequence of the deliberate blade mistuning even though the indices indicate only a minor degree of controllability. It is noticed that, for increasing velocities, the controllability indices of the modes 3 to 6 are decreasing while the level of controllability of the modes 1 and 2 are almost constant. This is due to the fact that the vibration coupling among disc and blades reduces. The controllability indices of the modes 1 and 2 tend to converge towards the same steady levels as for the tuned system of Fig. 4 . The observability indices show that the system are also, to some extend and at some velocities, observable from disc movement measurements. The observability indices of the first two modes are very similar to the values obtained for the tuned system shown in Fig. 4. Due to the decreasing vibration coupling, it is seen that the modes 3 to 6 become non-observable for increasing speeds. This implies that the rotational speed becomes of crucial importance while analysing the controllability and observability using only shaft actuation and sensing.

The levels of controllability and observability depend on the degree of mistuning. Figure 8 shows the controllability and observability indices of the least controllable and observable mode as functions of the percentage level of the blade tip mass mistuning. The mass of the $i$ th tip mass is given by $m_{t i}=(1+(i-1) \mu) m_{t 1}$ for $i=1,2,3,4$ where $\mu$ is the percentage of mistuning and $m_{t 1}$ is the tip mass of blade 1 . At first one would think, that increasing the level of blade mistuning would enhance the levels of controllability and observability. However, observing the indices it seems like the controllability tends to converge towards steady levels as the level of mistuning are increased towards "infinity". Increasing the degree of mistuning by means of adding more mass to the blades implies that the vibration coupling among disk and blades should be increased. On the other hand increasing the masses enhance the centrifugal stiffening of the blades having an opposing effect on the vibration coupling. In fact the controllability and observability maps show that a significant increase of such indices can be obtained by a minor mistuning, i.e. less than $1 \%$. Only a minor increase of the controllability can be achieved by a further increase of mistuning, i.e. 10 or $15 \%$. Of course, from the practical viewpoint, $20 \%$ of mass changes are far from reasonable mistuning ranges. Nevertheless, the graphics clarify some extreme cases of mistuning. The observability index becomes smaller with the increase of mistuning, achieving a minimum value that depends on the angular velocity and degree of mistuning. At the angular velocity of 600 RPM such a index attains the minimum value in the interval between $5 \%$ and $10 \%$ mistuning, which can be explained by the approximation of the veering ranges. Such behavior can also be detected at the angular velocity of 1200 RPM. It should be noticed that the controllability generally decreases as the angular velocity increases. Contrarily, the observability generally increases as a function of the angular velocity. This behavior can be intuitively explained: by increasing the angular velocity, the centrifugal forces, imposing the blade centrifugal stiffening effect, will increase quadratically with the angular velocity. That means, the differences among the blade natural frequencies will become bigger with the increase of angular velocity. Thus, the vibration modes (basic and parametric) will subsequently be more clear and observable. On the other hand, the quadratic increase of the centrifugal forces will lead to more "stiff" blades, what will require more controller effort in order to reduce the vibration levels. Thus, the controllability indices will decrease by increasing the angular velocity. Nevertheless, it is noticed that the controllability as well as observability indices are very small and their limits can be quantified. 


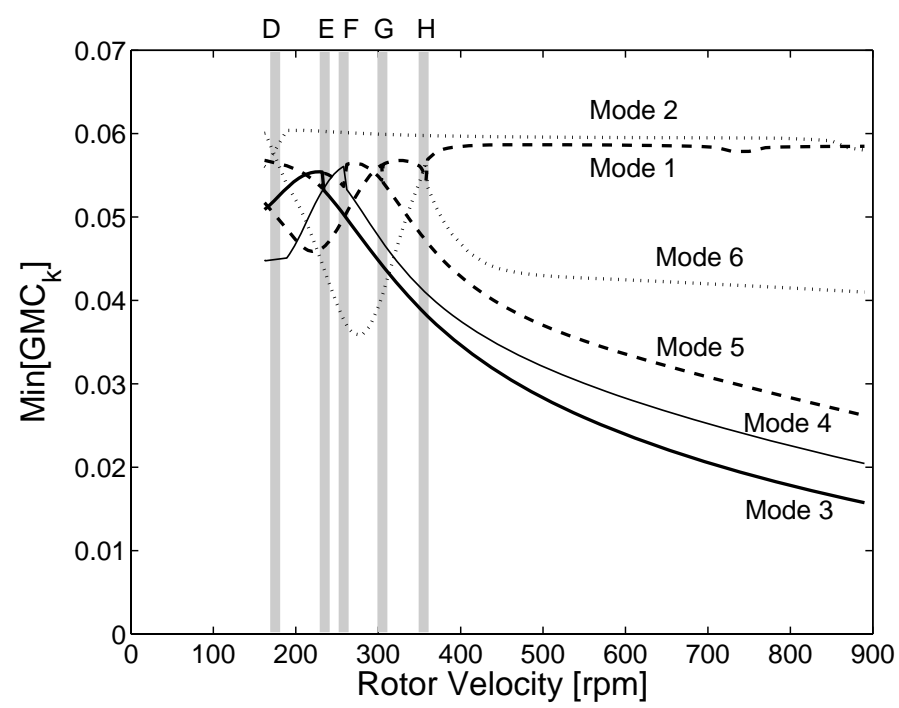

(a)

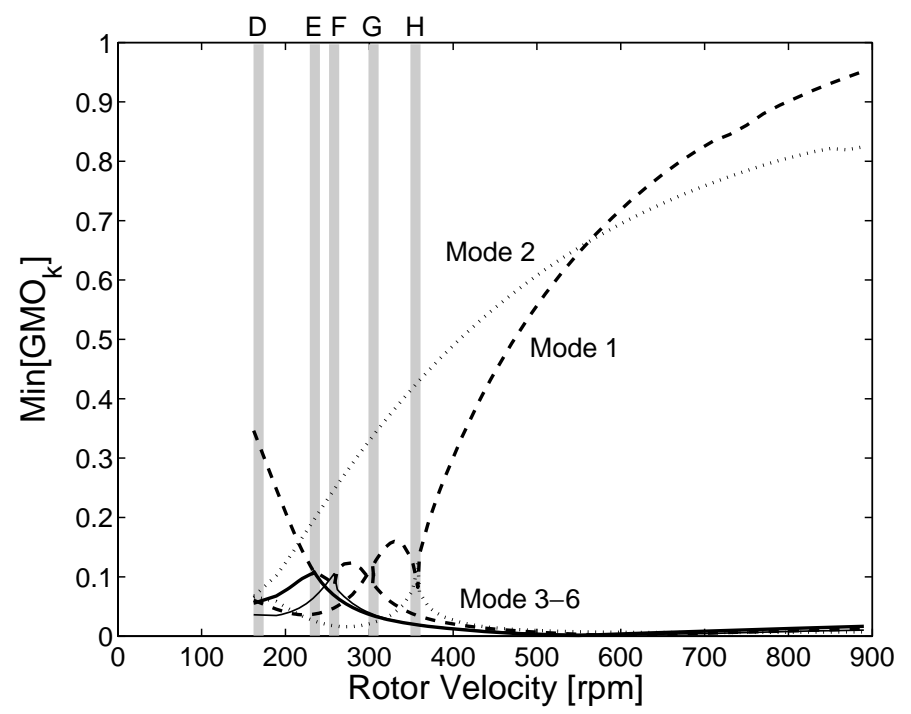

(b)

Fig. 7. Indices of modal controllability (a) and observability (b) for the mistuned rotor-blade system.

\subsection{Mistuned pattern analysis}

This far, the considered system has been mistuned by implementing four blades all with different geometrical parameters. However, other patterns of mistuning have also been studied. For instance implementing pairwise mistuning of opposite blades of the four bladed system, it can in fact be shown that the system become controllable and observable. Though it has to be emphasized that once the pairwise mistuned four bladed rotor is controllable and observable, it does not automatically imply that any pairwise mistuned system will be so as well. If the pairwise mistuning is introduced so that the opposing blades are identical, e.g. $m_{t 1}=m_{t 3}, m_{t 2}=m_{t 4}$ and $m_{t 1} \neq m_{t 2}$, then all four parametric mode shape components will relate to only two modes. Two blade modes will be associated with one backward and one forward rotating parametric mode component while the remaining two blade modes will be 


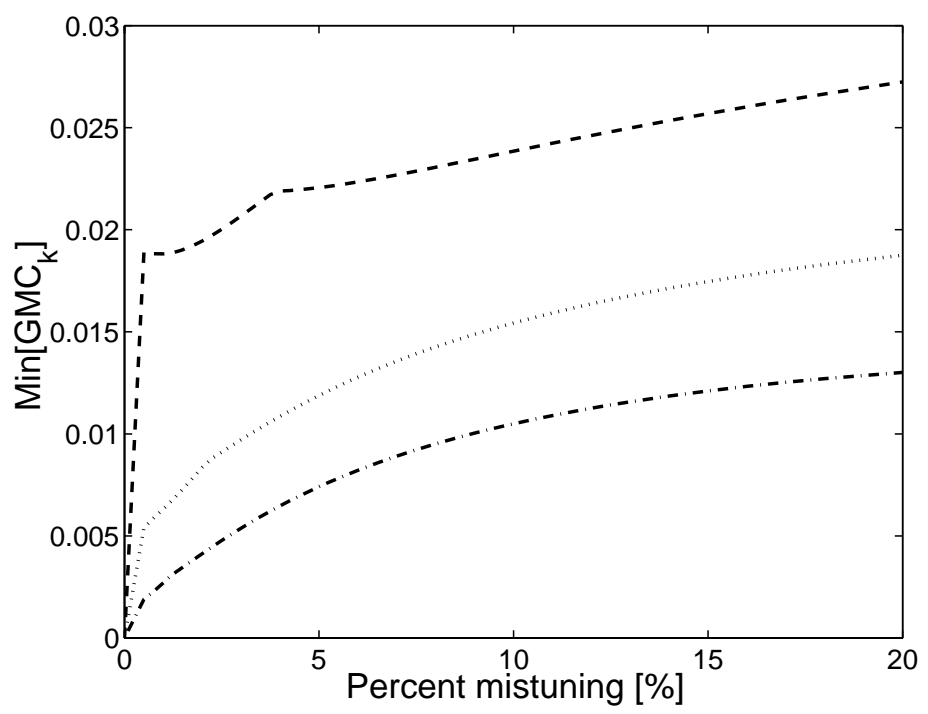

(a)

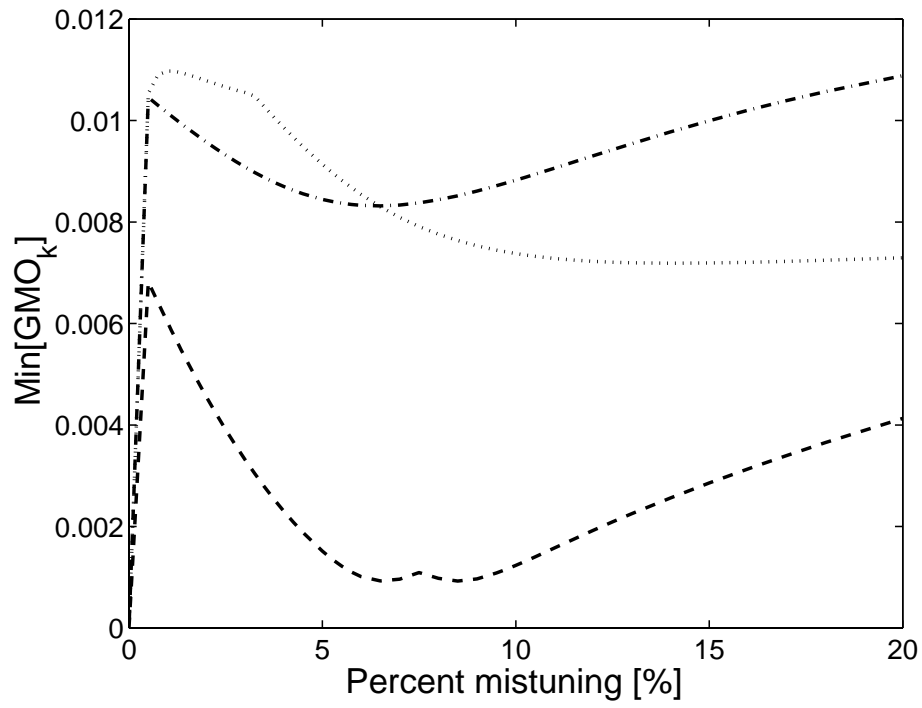

(b)

Fig. 8. Minimum index of modal controllability (a) and observability (b) as function of the degree of blade tip mass mistuning. $\Omega=600 \mathrm{rpm}$ $(--) ; \Omega=900 \mathrm{rpm}(.$.$) and \Omega=1200 \mathrm{rpm}(-\cdot-)$.

decoupled from rotor motion. Consequently, for such blade configuration two modes will still be non-controllable and non-observable.

Figure 9 shows the results of controllability analyses of various tuned and mistuned bladed rotor configurations with 3, 4 or 6 blades. The numbers indicate the dynamical characteristics of each blade so that blades with equal numbers are identical. The tuned systems in Figs 9(a), (d) and (h) are all non-controllable while the completely blade mistuned system in Figs 9(c), (g) and (m) make all blades controllable. Among the remaining configurations, the rotors (e), (i) and (k) will be controllable while (b), (f), (j) and (l) are non-controllable.

Of course the challenges of implementing mistuning pattern will always depend on the number of blades attached to the disc. In case of few blades, as wind turbines and helicopter blades, such implementation is not so complicated and can be carried out by adding or reducing blade mass and sequentially balancing. In case of many blades, the 


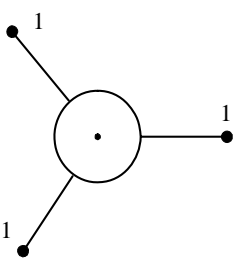

(a) Non-controllable

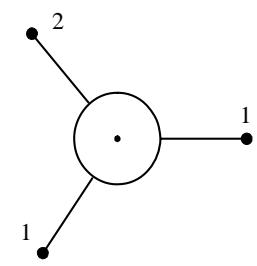

(b) Non-controllable

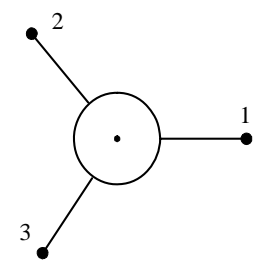

(c) Controllable

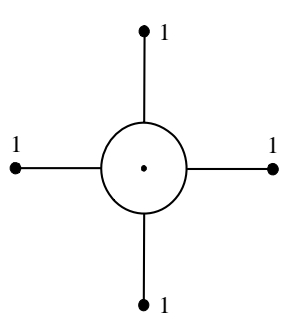

(d) Non-controllable

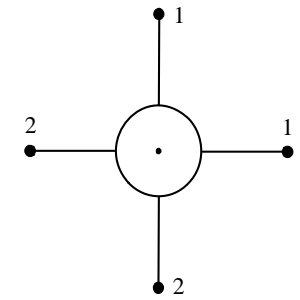

(e) Controllable

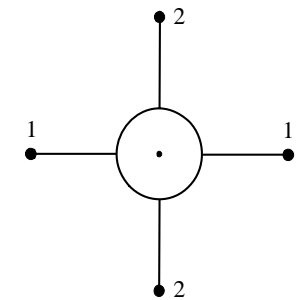

(f) Non-controllable

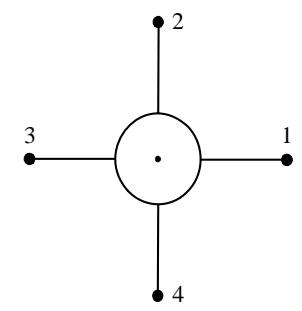

(g) Controllable

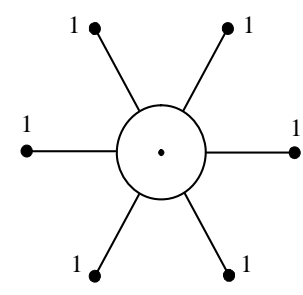

(h) Non-controllable

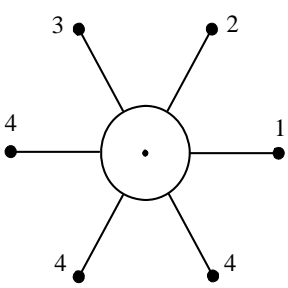

(k) Controllable

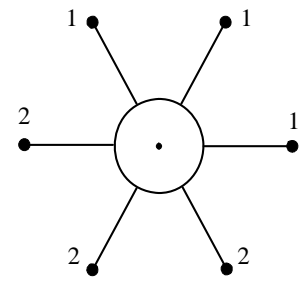

(i) Controllable

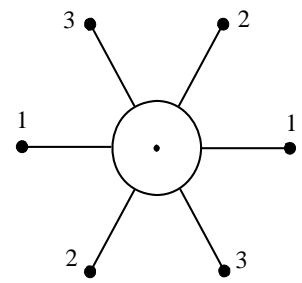

(j) Non-controllable

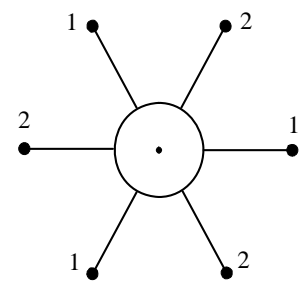

(l) Non-controllable

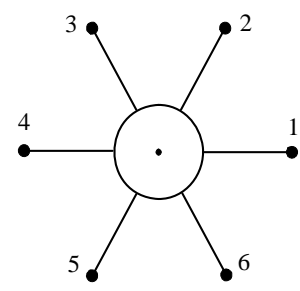

(m) Controllable

Fig. 9. Various patterns of mistuning for a three- four- and six-bladed rotor.

practical implementation of deliberate mistuning could be done by using different techniques, described in detail in $[3,6,7,14,23]$.

\section{Experimental control results}

To study the feasibility of rotor-blade coupled vibration control via shaft-based actuation, an active control scheme is applied to the experimental test facility. As it was described in the introduction, the active controller can be designed and implemented using one of two very different strategies, i.e. an absorbing strategy or a forcing strategy. The first one of these two strategies, the absorbing strategy, is based on suppressing blade vibrations by means of suppressing the vibrations of the rotor. The blade vibration energy can be absorbed via shaft vibration control due to the vibration coupling between rotor and blades, however, the use of such a strategy requires significant vibration coupling. The alternative forcing strategy is based on the principle of forcing the movement of the rotor 


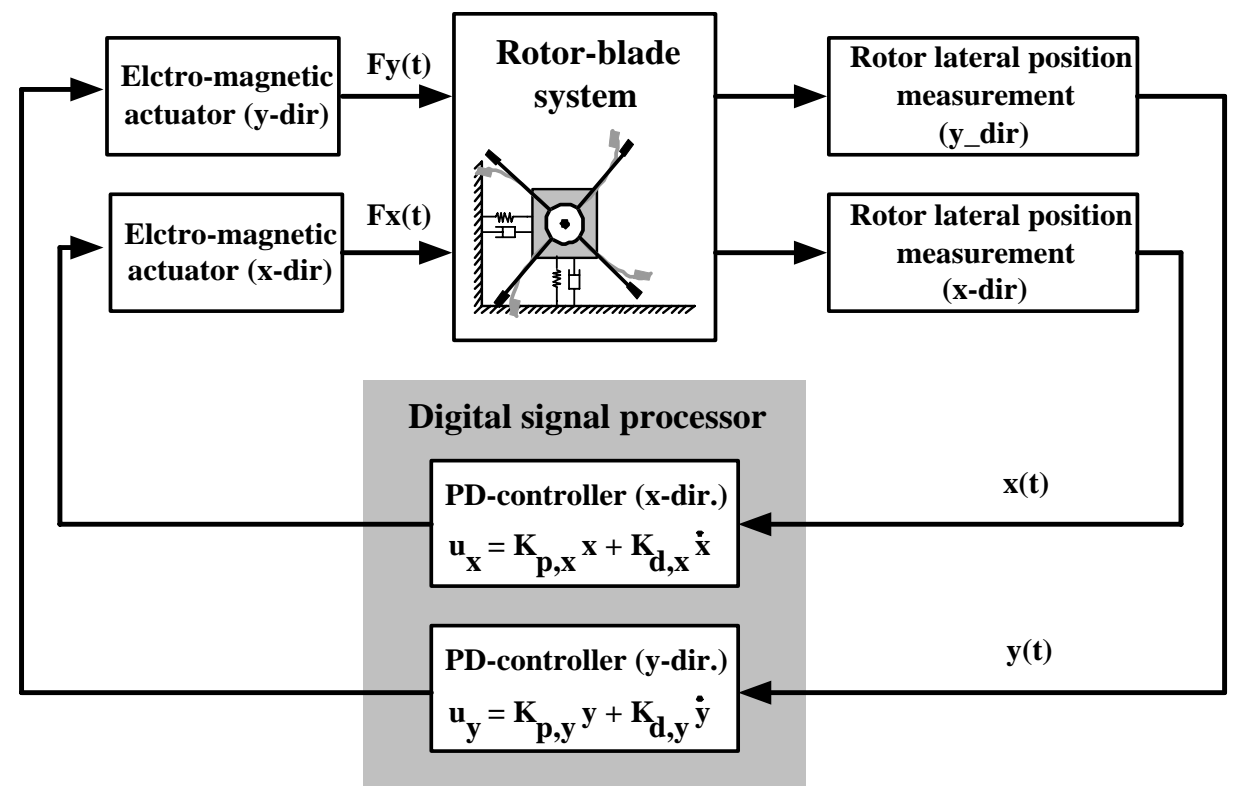

Fig. 10. Schematic drawing of the PD-controlled rotor-blade system.

in order to minimize the deflections of the blades. Such strategy will be applicable to systems with a low degree of vibration coupling but it will also inevitable mean that a time-variant control algorithm, encountering the time-variant distribution of inertia, has to be implemented. Such a strategy was adopted in [20,21] where a periodic modal state feedback controller was designed and implemented. However, several typical problems related to practical implementation of state feedback controllers relying heavily on quantitative model information were identified, i.e. lack of robustness and a high sensitivity to model uncertainties.

The system considered in this study presents significant coupling between rotor and blade motions. Therefore, the simpler control strategy of the two is adopted and a control scheme composed of two independent shaft-based PD-controllers is implemented. Figure 10 shows a schematic drawing of the control system setup. Rotor lateral movements are measured in two orthogonal directions and the sensed displacements are fed back into the two PD-controllers. The control signals are sent via power amplifiers to the electro-magnetic actuators.

The performance of the active control scheme is measured experimentally. Figure 11 shows experimentally measured frequency response functions of the rotor and blade motion for the active controlled rotor-blade system rotating at the constant velocity $\Omega=300 \mathrm{rpm}$ compared to the passively running system. Such responses reveal that a significant reduction of rotor as well as blade vibration levels is in fact achieved. The maximum blade vibration amplitude around $18 \mathrm{~Hz}$ is reduced by the factor $\approx 2.5$ and the rotor amplitude around $11 \mathrm{~Hz}$ is reduced by a similar factor.

\section{Conclusion}

In this paper, shaft-based active control of rotor-blade coupled vibrations has been studied. The modal controllability and observability were studied and the necessity of introducing deliberate blade mistuning in order to make all vibration modes controllable and observable, via the shaft-based actuation and sensing, was shown. Mistuning degrees of the order of less than $1 \%$ leads to significant increases of controllability and observability indices. Maximum values of such indices are also theoretically quantified. It is worth to emphasize that mistuning degree is as important as mistuning pattern in case of shaft-based actuation. Mistuned bladed disc with significant vibration coupling among rotor and blades must also obey some mistuning patterns, otherwise the blade motions can not be controlled by using only shaft-based actuation. The results reveal that the dynamical characteristics and the levels of controllability and observability change significantly as function of the angular velocity. More specifically, when 


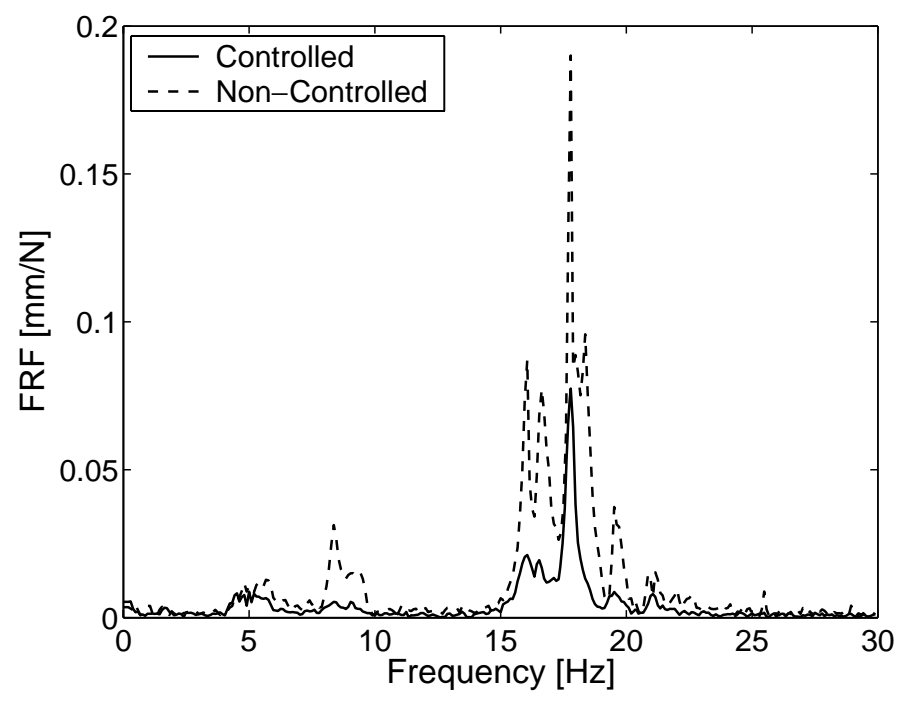

(a)

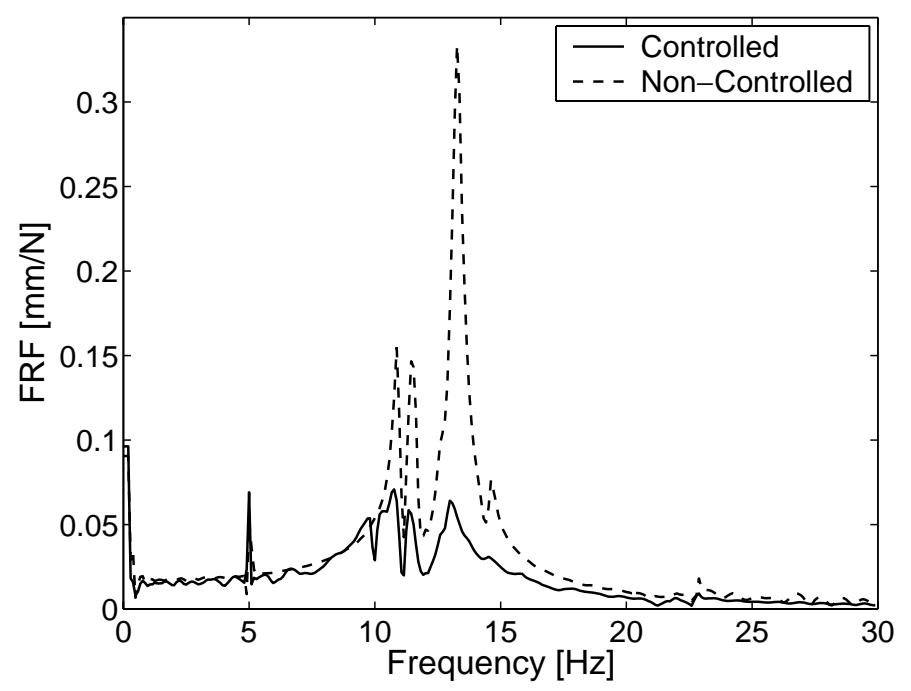

(b)

Fig. 11. Experimental frequency response functions of blade (a) and rotor lateral motion (b) for the non-controlled (- -) and actively controlled (一) rotor-blade system $(\Omega=300 \mathrm{rpm})$.

the bladed rotor rotates at certain velocities where parametric and basis vibration modes interact with themselves. The rotor-blade vibration coupling, resulting in such parametric vibrations, has to be taken into consideration when implementing active control. Furthermore, limits exist of how controllable and observable a bladed rotor can become via the introduction of deliberate blade mistuning degrees and patterns. In case of considering rotor tilt motion and gyroscopic effect, two additional natural frequencies would appear. Both basic natural frequencies would be dependent on the angular velocity and the rotor moment of inertia. This implies additional veering effect ranges, and, consequently, new controllability and observability maps. Finally, experimental results reveal the potential of controlling blade vibrations by using active controlled bearings, though, such control requires significant vibration coupling among rotor and blades in order to be efficient, although only this dynamic coupling is not sufficient to assure controllability. 


\section{References}

[1] A. Alizadeh, C. Ehmann, U. Schönhoff and R. Nordmann, Active Bearing of Rotors Utilizing Robust Controlled Piezo Actuators, Proceedings of ASME DETC'03, paper DETC2003/VIB-48850, Chicago, Illinois, USA, 2003.

[2] A.M.A. Hamdan and A.H. Nayfeh, Measure of Modal Controllability and Observability for First- and Second-Order Linear Systems, Journal of Guidance, Control and Dynamics 12(3) (1989), 421-428.

[3] B. Xiao, A.J. Rivas-Guerra and M.P. Mignolet, Maximum Amplification of Blade Response due to Mistuning in Multi-Degree-Of-Freedom Blade Models, Proceedings of ASME-IGTI Turbo Expo 2004, paper GT2004-54030, Vienna, Austria, 2004.

[4] B.O. Al-Bedoor, Blade Vibration Measurement in Turbo-Machinery: Current Status, The Shock and Vibration Digest 34(6) (2002), $455-461$.

[5] C.M. Saracho and I.F. Santos, Modal analysis in periodic, time-varying systems with emphasis to the coupling between flexible rotating beams and non-rotating flexible structures, Proceedings of the X International Symposium on Dynamic Problems of Mechanics, Sao Paulo, Brazil, 2003, 399-404.

[6] E. Capiez-Lernout and C. Soize, Specifying Manufacturing Tolerances for a Given Amplification Factor: A Nonparametric Probabilistic Methodolody, Proceedings of ASME-IGTI Turbo Expo 2003, paper GT2003-38050, Atlanta, USA, 2003.

[7] F. Götting, W. Sextro, L. Panning and K. Popp, Systematic Mistuning of Bladed Disk Assemblies with Friction Contacts, Proceedings of ASME-IGTI Turbo Expo 2004, paper GT2004-53310, Vienna, Austria, 2004.

[8] G. Schweitzer, Magnetic Bearings as a Component of Smart Rotating Machinery, Proceedings of The Fifth International Conference on Rotor Dynamics, Darmstadt, Germany, 1998, 3-15.

[9] G. Szász, G.T. Flowers and R.J. Hartfield, Hub-Based Vibration Control of Multiple Rotating Airfoils, Journal of Propulsion and Power 16(6) (2000), 1155-1163.

[10] G. Szász and G.T. Flowers, Time Periodic Control of a Bladed Disk Assembly Using Shaft Based Actuation, Journal of Vibration and Acoustics 123 (2001), 395-401.

[11] G. Szász and G.T. Flowers, Vibration Suppression in Bladed-Disk Assemblies With Deliberate Mistuning via Magnetic Bearings, Journal of Vibration and Control 6 (2000), 903-921.

[12] I.F. Santos, C.M. Saracho, J.T. Smith and J. Eiland, Contribution to Experimental Validation of Linear and Non-linear Dynamic Models for Representing Rotor-Blade Parametric Vibrations, Journal of Sound and Vibration 271(3-5) (2004), 883-904.

[13] I.F. Santos and A. Scalabrin, Control System Design for Active Lubrication with Theoretical and Experimental Examples, ASME Journal of Engineering for Gas Turbines and Power 125(1) (2003), 75-80.

[14] J.A. Kenyon and J.H. Griffin, Experimental Demonstration of Maximum Mistuned Bladed Disk Forced Response, Proceedings of ASMEIGTI Turbo Expo 2003, paper GT2003-38060, Atlanta, USA, 2003.

[15] J.A. Kenyon, J.H. Griffin and N.E. Kim, Sensitivity of Tuned Bladed Disk Response to Frequency Veering, Proceedings of ASME/IGTI Turbo Expo 2004, paper GT2004-53280, Vienna, Austria, 2004.

[16] J. Althaus and H. Ulbrich, A Fast Hydraulic Actuator for Active Vibration Control, Proceedings of The Fifth International Conference on Vibrations in Rotating Machinery, Bath, England, 1992, 141-148.

[17] J. Xu and R. Gasch, Modale Behandlung linearer periodisch zeitvarianter Bewegungsgleichungen, Archive of Applied Mechanics 65(3) (1995), 178-193, (in German).

[18] M. Zielinski and G. Ziller, Noncontact Vibration Measurements on Compressor Rotor Blades, Measurement Science Technology 11 (2000), 847-856.

[19] R.H. Christensen, Active Vibration Control of Rotor-Blade Systems - Theory and Experiment, Ph.D. thesis, ISBN 87-90130-05-7, Technical University of Denmark, 2005.

[20] R.H. Christensen and I.F. Santos, A Study of Active Rotor-Blade Vibration Control Using Electro-Magnetic Actuation - Part 1: Theory, Proceedings of ASME-IGTI Turbo Expo 2004, paper GT2004-53509, Vienna, Austria, 2004.

[21] R.H. Christensen and I.F. Santos, A Study of Active Rotor-Blade Vibration Control Using Electro-Magnetic Actuation-Part 2: Experiments, Proceedings of ASME-IGTI Turbo Expo 2004, paper GT2004-53512, Vienna, Austria, 2004.

[22] R.H. Christensen and I.F. Santos, Design of Active Controlled Rotor-Blade Systems Based on Time-Variant Modal Analysis, Journal of Sound and Vibration 280 (2005), 863-882.

[23] S.H. Lim, M.P. Castanier and C. Pierre, Intentional Mistuning Design Space Reduction Based on Vibration Energy Flow in Bladed Disks, Proceedings of ASME-IGTI Turbo Expo 2004, paper GT2004-53873, Vienna, Austria, 2004. 

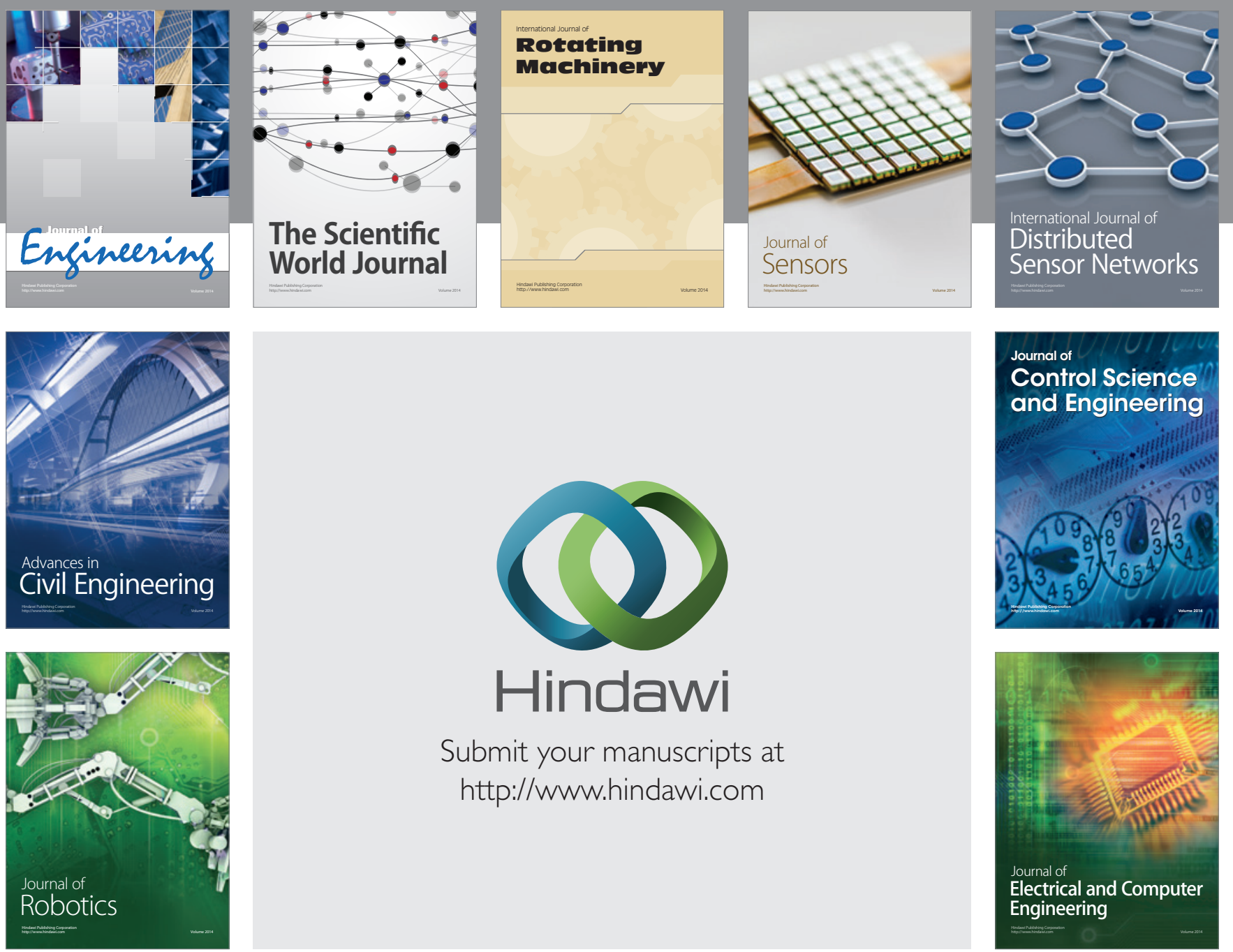

Submit your manuscripts at

http://www.hindawi.com
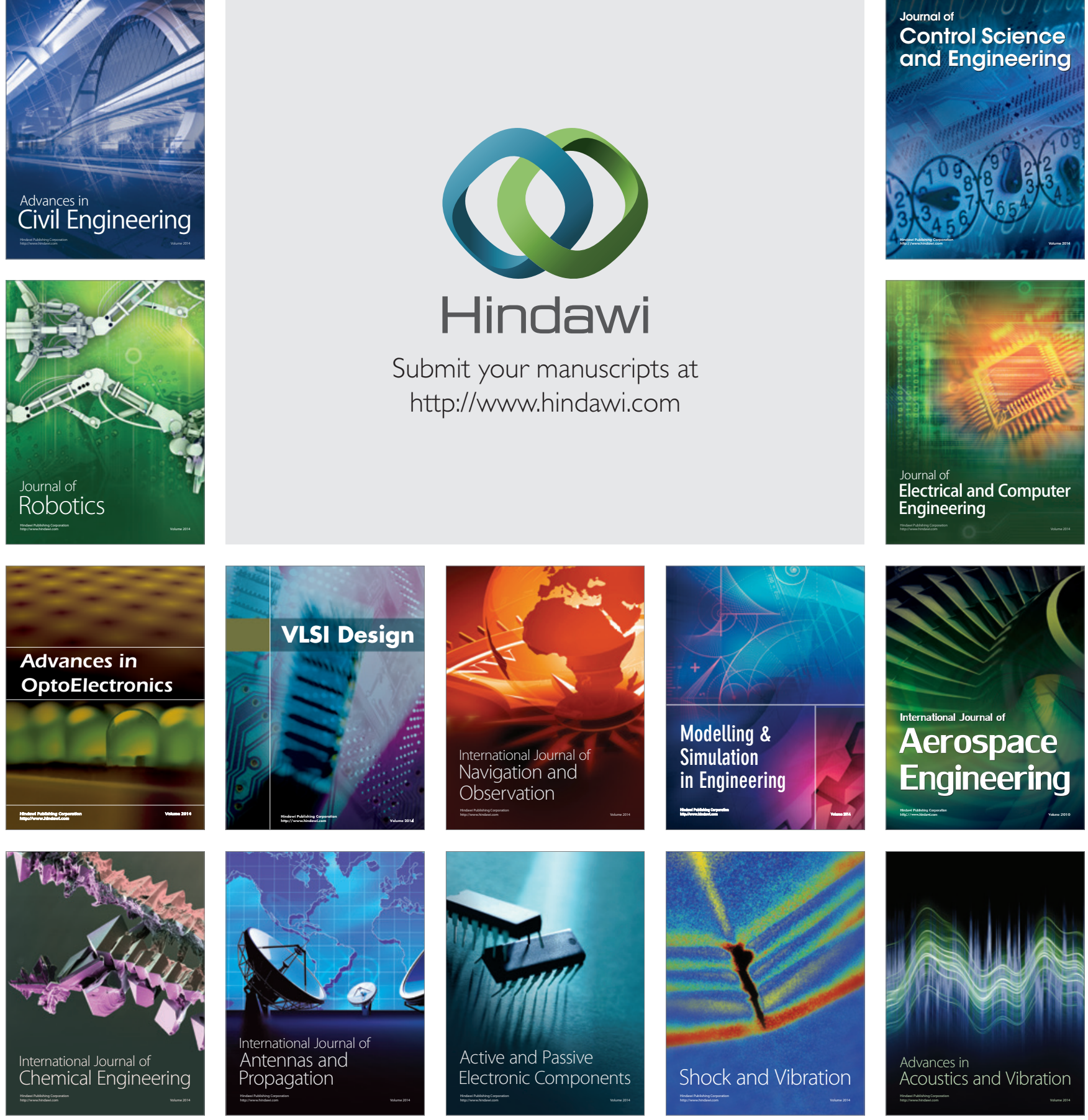